Nanotechnology Vol 15, pp.802-806 (2004)

\title{
VISIBLE AND NEAR IR PHOTOLUMINESCENT RESPONSE OF nc-Si:Er THIN FILMS PRODUCED BY rf SPUTTERING
}

\author{
M.F. Cerqueira $^{1}$, T. Monteiro ${ }^{2}$, M.V. Stepikhova ${ }^{3}$, M. Losurdo ${ }^{4}$, M.J. Soares ${ }^{2}$, \\ Isabel Gomes $^{1}$ \\ ${ }^{1}$ Departamento de Física, Universidade do Minho, Campus de Gualtar 4710-057 Braga, \\ Portugal. \\ ${ }^{2}$ Departamento de Física, Universidade de Aveiro, Campus de Santiago 3700 Aveiro, \\ Portugal \\ ${ }^{3}$ Institute for Physics of Microstrucutres RAS, 603600 Nizhnij Novgorod GSP-105 \\ Russia Plasma Chemistry Research Center, CNR, Via Orbona, 4, 70126 Bari Italy \\ ${ }^{4}$ Plasma Chemistry Research Center, CNR, Via Orbona, 4, 70126 Bari Italy
}

\begin{abstract}
In this contribution we present the Visible and near IR photoluminescence (PL) analysis of Er doped nanocrystalline silicon thin films produced by rf magnetron sputtering method. Efficient photoluminescence was observed in these structures in both visible and $1.54 \mu \mathrm{m}$ wavelength regions. We show the strong influence of the presence of nanocrystalline fraction in films on their luminescence efficiency at $1.54 \mu \mathrm{m}$ that has been studied on the series of specially prepared samples with the different crystallinity, i.e. percentage and sizes of $\mathrm{Si}$ nanocrystals. The mechanism involved in the visible photoluminescence of highly a crystalline nc-Si:H consisting of about $7 \mathrm{~nm}$ silicon nanocrystals embedded in an amorphous matrix is discussed.
\end{abstract}

\section{Introduction}

Semiconductor nano-structures with $<10 \mathrm{~nm}$ typical size, are of considerable current interest because of the new physics involved and potential device applications. Among them Er doped nc-Si samples are well known to be suitable for optoelectronic applications mainly in the mid infrared region, where the emission from the first excited state to the ground state of $\mathrm{Er}^{3+}$ ion is observed near $1.54 \mu \mathrm{m}[1,2$ and references therein].

In this contribution we show that Er-doped nc-Si:H thin films emitting at room temperature in the visible and IR range can be deposited by the reactive magnetron sputtering method. We discuss the role of nanocrystalline fraction in films on their luminescence efficiency at $1.54 \mu \mathrm{m}$ and report for the first time on the visible 
luminescence properties of these samples investigated under low excitation conditions. Steady state photoluminescence (PL), time resolved spectroscopy and decay time measurements carried out in the temperature range between $14 \mathrm{~K}$ and room temperature (RT) will be presented.

\section{Experimental details}

Erbium doped nanocrystalline silicon thin films were grown by r.f. reactive magnetron sputtering in an $\mathrm{Ar} / \mathrm{H}_{2}$ atmosphere on ordinary glass substrates under several different conditions (RF power, temperature, Er content and gas mixture composition) applying a procedure similar to that used for the preparation of undoped $\mu \mathrm{c}-\mathrm{Si}: \mathrm{H}$ films [3]. The target used was a c-Si of high purity (99.99\%). The erbium was placed in a low erosion area on the silicon target, in order to keep the moderate rate of Er impurity. The substrate-target distance was fixed at $55 \mathrm{~mm}$.

Applying this method samples with different structural parameters, i.e., different crystalline fraction and grain sizes can be produced. Parameters of some samples grown at specified conditions to obtain samples with different crystallinity are shown in Tables 1 and 2. Details of chemical composition, structural characterisation, microstructure analysis and films anatomy of these samples were described elsewhere [4].

Table 1: Growth conditions for erbium doped nanocrystalline silicon thin films

\begin{tabular}{|c|c|c|c|}
\hline Sample & $\begin{array}{c}\text { Temperature } \\
\left({ }^{\circ} \mathrm{C}\right)\end{array}$ & $\begin{array}{c}\text { RF power } \\
(\mathrm{W})\end{array}$ & $\mathrm{R}_{\mathrm{H}}$ \\
\hline Er22 & 400 & 80 & 0.63 \\
\hline Er19 & 200 & 80 & 0.63 \\
\hline Er24 & 50 & 80 & 0.63 \\
\hline Er28 & 200 & 150 & 0.37 \\
\hline Er33 & 25 & 80 & 0.63 \\
\hline
\end{tabular}

$N B: R_{H}=p_{2} /\left(p H_{2}+p A r\right)$ is the hydrogen fraction.

Photoluminescence (PL) measurements in the near IR range have been performed with a Brucker 66V Fourier-transform spectrometer, where $514.5 \mathrm{~nm}$ line of an Ar laser was used for the excitation. The signal was detected with a North-Coast EO-817 liquid nitrogen cooled germanium detector. In the visible, the samples were excited with 325 $\mathrm{nm}$ line of $\mathrm{cw} \mathrm{He}-\mathrm{Cd}$ laser with the excitation power density kept less than $0.6 \mathrm{~W} . \mathrm{cm}^{-2}$. The PL signal was measured between 14K and RT using a closed cycle helium cryostat. Time resolved spectra were performed with the pulsed Xe lamp (50 $\mathrm{mW})$ as an excitation source and a boxcar system for detection (time range from hundred of $\mu$ s to 
seconds). The visible PL was dispersed by a Spex 1704 monochromator with the $1200 \mathrm{~mm}^{-1}$ grating and detected by a cooled Hamamatsu R928 photomultiplier. All spectra presented were corrected taking into account the spectral sensitivity of the detection system.

Table 2: Elements content, thickness and structural parameters of nc-Si:Er samples.

\begin{tabular}{|c|c|c|c|c|c|c|c|c|c|}
\hline Sample & $\begin{array}{c}\mathrm{Er} \\
(\%)\end{array}$ & $\begin{array}{c}\mathrm{Si} \\
(\%)\end{array}$ & $\begin{array}{c}\mathrm{O} \\
(\%)\end{array}$ & $\begin{array}{c}\mathrm{H} \\
(\%)\end{array}$ & $\begin{array}{c}\mathrm{d} \\
(\mathrm{nm})\end{array}$ & $\begin{array}{c}\mathrm{D}_{\mathrm{X}} \\
(\mathrm{nm})\end{array}$ & $\begin{array}{c}\mathrm{D}_{\mathrm{R}} \\
(\mathrm{nm})\end{array}$ & $\begin{array}{c}\mathrm{C}_{\mathrm{R}} \\
(\%)\end{array}$ & $\begin{array}{c}\mathrm{SE} \\
\text { data }\end{array}$ \\
\hline Er22 & 0.10 & 71.7 & 8.8 & 17.6 & 2089 & 7.0 & 7.9 & 65 & $87 \% \mu \mathrm{c}-\mathrm{Si}$ \\
\hline Er19 & 0.12 & 62 & 34 & 23 & 483 & 5.7 & 6.5 & 43 & $31 \% \mu \mathrm{c}-\mathrm{Si}$ \\
\hline Er24 & 0.17 & 56.5 & 17.6 & 25.4 & 538 & 3.9 & 5.5 & 23 & $25 \% \mu \mathrm{c}-\mathrm{Si}$ \\
\hline Er28 & 0.11 & 60.9 & 2.9 & 34.3 & 1295 & - & - & 0 & $10 \% \mathrm{nc}-\mathrm{Si}$ \\
\hline Er33 & 0.02 & 73.4 & $<1$ & 25.8 & 1499 & - & - & 0 & $38 \% \mathrm{nc}-\mathrm{Si}$ \\
\hline
\end{tabular}

NB: $D$ - average crystal size ( $R$ - Raman spectroscopy, $X$-XRD analysis); $C_{R}$ - crystalline volume fraction determined by Raman spectroscopy, $d$ - film thickness, SE data - data of the film anatomy analysis performed by spectroscopic ellipsometry (for more details see [5]).

\section{Results and discussion}

\section{$P L$ response of $n c-S i:$ Er samples in near IR range}

Near IR photoluminescence spectra measured at $77 \mathrm{~K}$ in nc-Si:Er samples are shown in Fig. 1. The spectra of these samples show the luminescence peak at $1.54 \mu \mathrm{m}$ related with the itra-atomic $\left({ }^{4} \mathrm{I}_{13 / 2} \rightarrow{ }^{4} \mathrm{I}_{15 / 2}\right)$ transitions of $\mathrm{Er}^{3+}$ ions. The most intense Er-related PL was observed in samples with the lowest crystallinity that have according our TEM measurements $\mathrm{Si}$ nanocrystalline inclusions of the sizes less than $3 \mathrm{~nm}$ [6]. Being relatively broad in low crystalline sample (sample Er24 in Fig. 1) with the maximum at $6500 \mathrm{~cm}^{-1}$ and a characteristic shoulder at around $6457 \mathrm{~cm}^{-1}$, like for the Er in a glasslike and amorphous materials [7] The Er-related spectrum transforms in highly crystalline films (sample Er22) in the spectrum with a fine line structure giving the evidence for the incorporation of Er ions in regular crystalline surroundings. However the increase of crystallinity in the samples results in the strong quenching of Er related photoluminescence. So, for the samples with the similar Er atomic percentage $(0.1 \div 0.17$ $\%$, as estimated by RBS), PL intensity reduces by more than an order of magnitude with the increasing of crystalline fraction from $23 \%$ to $65 \%$ (and crystallite sizes from 5.5 to $7.9 \mathrm{~nm}$, see the insert in Fig.2). For the sample with the lowest crystallinity (sample Er33 in Fig. 1), PL intensity exceeds that for highly crystalline samples (sample Er22) by about two orders of magnitude, this is despite the lower Er content in highly crystalline film (see Table 2). Moreover, at room temperature the photoluminescence 
was practically undetectable in highly crystalline samples. As we have discussed earlier [8], this luminescent behaviour of nc-Si:Er thin films can be described as a result of sensitizing effect of Er ions by $\mathrm{Si}$ nanocrystals of small sizes $(<3 \mathrm{~nm})$ that is related with the enhancement of excitation probability for Er ions being activated by the recombination of excitons spatially confined in Si nanocrystals. Similar situation was observed also by M. Fujii et al. [9], who has studied the correlation between Er-related $\mathrm{PL}$ intensity and the nc-Si grain sizes in Si-nc containing $\mathrm{SiO}_{2}$ films doped with Er. These authors have obtained strong, by about two orders of magnitude, increase of Er PL intensity with the lowering of Si-nc grain sizes from 3.8 to $2.7 \mathrm{~nm}$. Starting with these sizes, the remarkable bandgap widening and strong increase of recombination rate for excitons confined in Si nanocrystals should be expected [10].

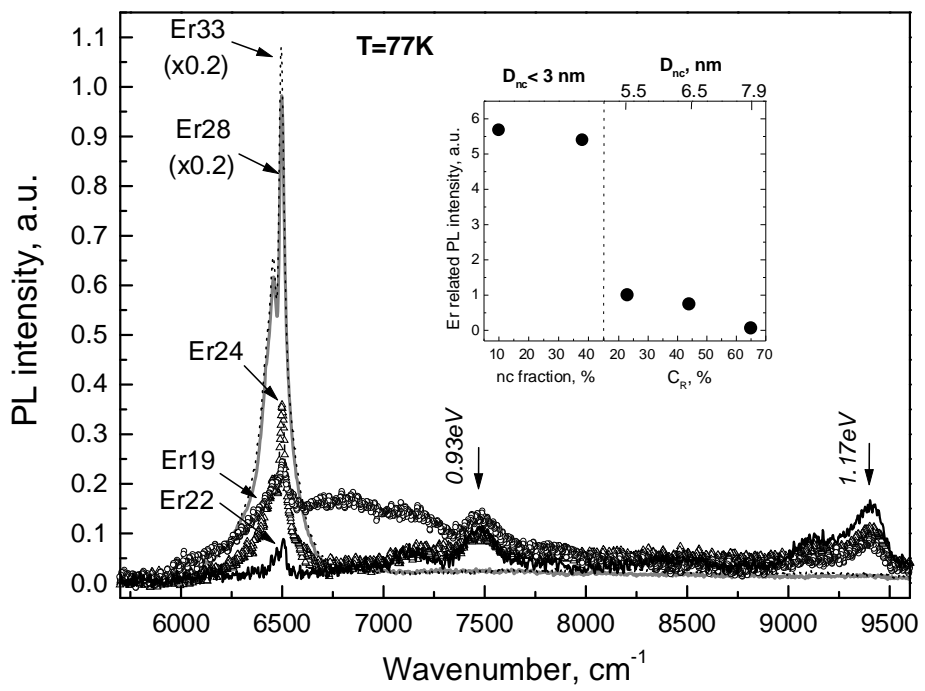

Fig. 1 - PL spectra of nc-Si:Er samples with the different content of crystalline fraction. Inserts show the correlation between the PL intensity (Er-related peak) and the presence of crystalline fraction in nc-Si:Er samples. Right part of this insert shows the situation for highly crystalline samples according to XRD and Raman analysis (the $C_{R}$ and $D_{R}$ values on the bottom and top axis correspond to the Raman data). On the left part, PL intensities for the samples with the nanocrystallite fraction with 1-3 nm crystallite grain sizes are presented, where the values for $\mathrm{nc}$ fraction are taken from SE data. The luminescence intensities at $1.54 \mu \mathrm{m}$ in this insert are normalised to the films thickness.

\section{$P L$ response of $n c-S i:$ Er samples in the visible range}

Strong visible PL has been observed in nc-Si:Er samples with the less pronounced quenching up to room temperature. Here we emphasize the studies carried out on a highly crystalline sample with the crystalliny of about 65\% (sample Er22 in Table 1 and 2).

Figure 2a) shows the temperature dependence of the steady state PL spectra obtained under low excitation density in our highly crystalline nc-Si:Er sample which consists of 
nanocrystals with a diameter of $7.9 \mathrm{~nm}$ embedded in amorphous silicon matrix. For this sample, the visible PL has been clearly detected in the range from 1.45 to $3.0 \mathrm{eV}$.

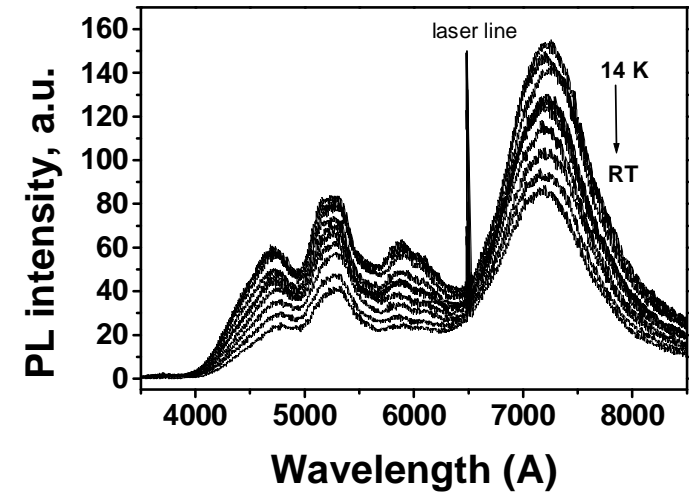

a)

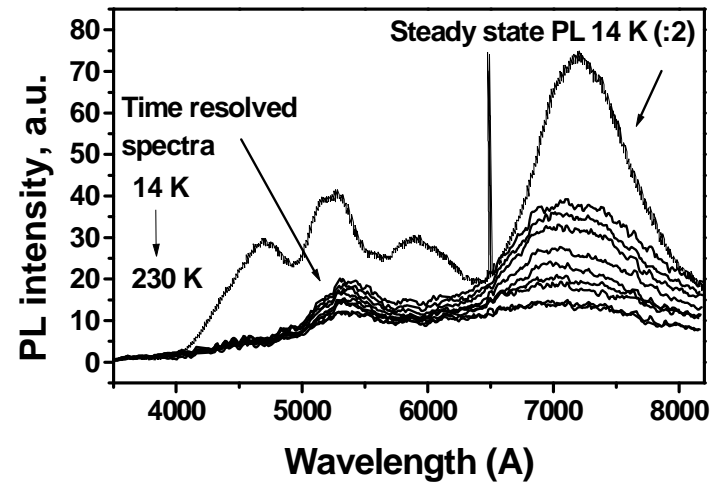

b)

Fig. 2 - a) Steady state PL spectra obtained under He-Cd laser excitation (excitation density $<0.6 \mathrm{~W} / \mathrm{cm}^{2}$ ). The spectra were measured in the temperature range from $14 \mathrm{~K}$ to room temperature. b) Comparison between $14 \mathrm{~K}$ steady state PL and time resolved spectra measured with the time delay of $0.1 \mathrm{~ms}$ and time window of $15 \mathrm{~ms}$ after the lamp pulse.

The observed spectra are dominated by a broad red emission band peaked near $717 \mathrm{~nm}$ $(1.73 \mathrm{eV})$. Minor intensity emission bands are also observed on the high-energy side with the main maxima close to $590 \mathrm{~nm}(2.1 \mathrm{eV}), 525 \mathrm{~nm}(2.36 \mathrm{eV})$ and $470 \mathrm{~nm}(2.64$ $\mathrm{eV})$. The spectrum obtained at $14 \mathrm{~K}$ can be fitted to Gaussian-shaped bands peaked at the main maxima with the FWHM (full widths at half maximum) values of $187 \mathrm{meV}$, $270 \mathrm{meV}, 145 \mathrm{meV}$ and $360 \mathrm{meV}$ for the $1.73 \mathrm{eV}, 2.1 \mathrm{eV}, 2.36 \mathrm{eV}$ and $2.64 \mathrm{eV}$ bands, respectively. The FWHMs for $1.7 \mathrm{eV}$ and $2.36 \mathrm{eV}$ emission bands in our experiment agree very well with those determined recently for the emission bands of isolated silicon quantum dots [11]. As one can see, PL intensity for all of the emission bands measured in our conditions decreases only by about $50 \%$ in the temperature range from $14 \mathrm{~K}$ to RT.

The comparison between the $14 \mathrm{~K}$ steady state PL and time resolved luminescence spectra obtained with a time delay of $0.1 \mathrm{~ms}$ and time window of $15 \mathrm{~ms}$ after the pulse excitation is presented in Figure 2b). The observed data under low excitation pump put in evidence the slow character of the narrower $1.73 \mathrm{eV}$ and $2.36 \mathrm{eV}$ emission bands while the $2.64 \mathrm{eV}$ and $2.1 \mathrm{eV}$ emission bands decay faster than the time response of the set up used. 
The photoluminescence transients (performed for the maxima of red $-1.73 \mathrm{eV}$, and yellow $-2.36 \mathrm{eV}$ bands) measured as a function of the delay time after the lamp pulse, are presented in Figure 3.

For both emission bands we observed the non-exponential decay of PL signal, which can be deconvoluted into two fast and slow exponential components. Here it should be mentioned that the time resolved spectroscopy performed with different delay times and time windows did not allow for any spectral resolution associated with these two decay components.
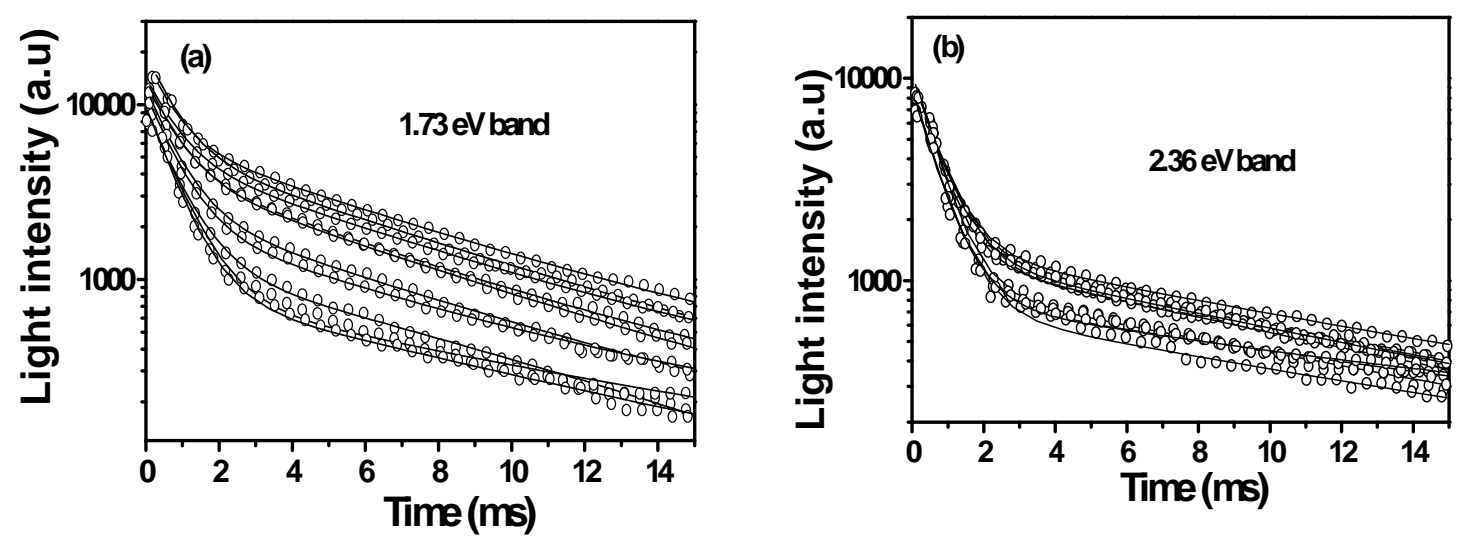

Fig. 3 - 14K to RT temperature dependence of the luminescence decay measured for: (a) 1.73 $\mathrm{eV}$ and (b) $2.36 \mathrm{eV}$ emission bands.

The decay times of the two components as a function of temperature are depicted in Figure 4. It can be inferred from the Figures 3 and 4 that both decays are almost temperature independent in the whole investigated temperature range. We found for the red and yellow emission bands the mean fast decay components of $0.74 \mathrm{~ms}$ and $0.68 \mathrm{~ms}$ and slow decay components of $6.8 \mathrm{~ms}$ and $11.6 \mathrm{~ms}$, respectively. 


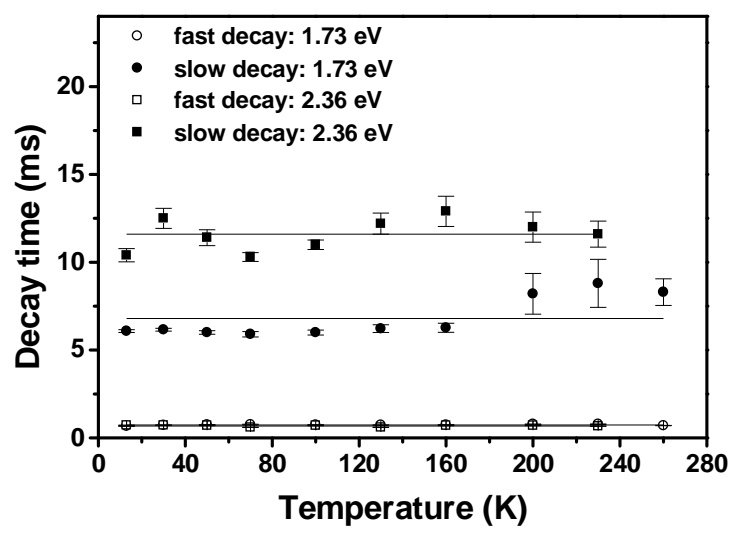

Fig. 4 - Temperature dependence of the decay times measured for the red $(1.73 \mathrm{eV})$ and yellow $(2.36 \mathrm{eV})$ luminescence bands.

One can assume that the bands at 1.73 and $2.36 \mathrm{eV}$ in visible response of nc-Si:Er films are related with the exciton recombination confined in Si nanocrystals. As we have mentioned above, FWHMs for these emission bands correlate very well with those determined for the emission bands of isolated silicon nanocrystals. Indeed, according to the model of excitonic recombination in nanocrystalline $\mathrm{Si}$ structures the decay times for excited (triplet) - ground (singlet) state transitions in Si nanocrystals should lie in millisecond time scale at low temperatures $[12,13]$. With the increasing temperature the fast (microsecond) component appears in PL decay that is related with the population of high-energy (singlet) excitonic state splited by the electron-hole exchange interaction. Both time constants should strongly depend on the nanocrystal sizes, moreover the temperature, at which the fast transitions from the upper singlet state became allowed is also nanocrystal size determined $[12,13]$. The time constants (i.e. the radiative rates) for excitonic transitions should decrease by more than an order of magnitude with the decreasing of nanocrystal sizes, this decrease for the faster decay times (accounting for transitions from the upper singlet states) must be more pronounced.

As it has been shown, the PL decays for 1.73 and $2.36 \mathrm{eV}$ bands in our experiment can be also well fitted with two components in millisecond and microsecond ranges, however the faster ones are practically identical for both bands and appear already at low temperature. More plausible explanation for that is the relation of these fast (microsecond) decays with some non-radiatiative recombination processes occuring with the participation of defects in nanocrystals or on the nanocrystal grain boundaries. This means that only the slow recombination components could be addressed to the 
transitions from the excited (triplet) to ground state excitonic transitions in $\mathrm{Si}$ nanocrystals, whereas the fast decay components identified describe the non-radiative processes. Indeed, accounting for that we can explain the absence of spectral changes in the time resolved PL spectra measured with different time delays and time windows and also explain the decrease of PL intensity observed with the increasing temperature in our experiment upon practically no changes in the time constants. One can show that the increase of temperature in our case leads to the variation of the ratio between the fast and slow decay components $\left(\mathrm{I}_{\text {fas }} / \mathrm{I}_{\text {slow }}\right)$ for the two visible PL bands (see Fig. 5a), at that the contribution from the fast components increases in the whole transient range with temperature. It means that the contribution of nonradiative processes increases with the temperature. So, at $14 \mathrm{~K}$ we have the ratio between fast and slow decay components $\left(\mathrm{I}_{\text {fast }} / \mathrm{I}_{\text {slow }}\right) 2$ and 7 for $1.7 \mathrm{eV}$ and $2.36 \mathrm{eV}$ bands, respectively, but at room temperature this ratio changes to 10 and 12 , respectively. The temperature dependence of steady state PL intensity and the extrapolation of PL intensity through these two slow and fast recombination components $\left(I_{P L}=I_{\text {fast }} \tau_{\text {fast }}+I_{\text {slow }} \tau_{\text {slow }} ; \tau\right.$ - decay times $)$ are presented in Figure 5b). As one can see, this model taking into account the participation of two mechanisms (excitonic, nanocrystal related and non-radiative ones) describes quite well the overall behavior of visible PL in our samples. One open question here is relation between the slow time constants obtained for $2.36 \mathrm{eV}$ and $1.73 \mathrm{eV}$ bands. We observe the shorter time constants $(\sim 6.8 \mathrm{~ms})$ for the low energy PL band and the longer ones $(\sim 11.6 \mathrm{~ms})$ for high-energy band.

\section{Conclusion}

In this contribution we have shown that efficient photoluminescence was observed in Er doped nc-Si:H thin films in both visible and $1.54 \mu \mathrm{m}$ wavelength regions.

The strong influence of Si nanocrystals sizes on the films luminescent properties at 1.54 $\mu \mathrm{m}$ has been observed, where the most intense Er photoluminescence obtained relates to the low crystalline films with the nanocrystal grain sizes less than $3 \mathrm{~nm}$.

From the study of the temperature dependence of PL and time resolved PL of visible bands observed in a highly crystalline nc-Si:Er sample it is inferred that slow and longlived luminescence in nc-Si is present. Both decay rates are constant in a temperature range for which decrease in PL intensity could be observed. We have shown that the slow recombination components could be addressed to the transitions from the excited 
(triplet) to ground state excitonic transitions in Si nanocrystals, whereas the fast decay components identified describe the non-radiative processes.

In order to establish a complete model for the recombination processes further experimental analysis must be realized, such as ODMR.

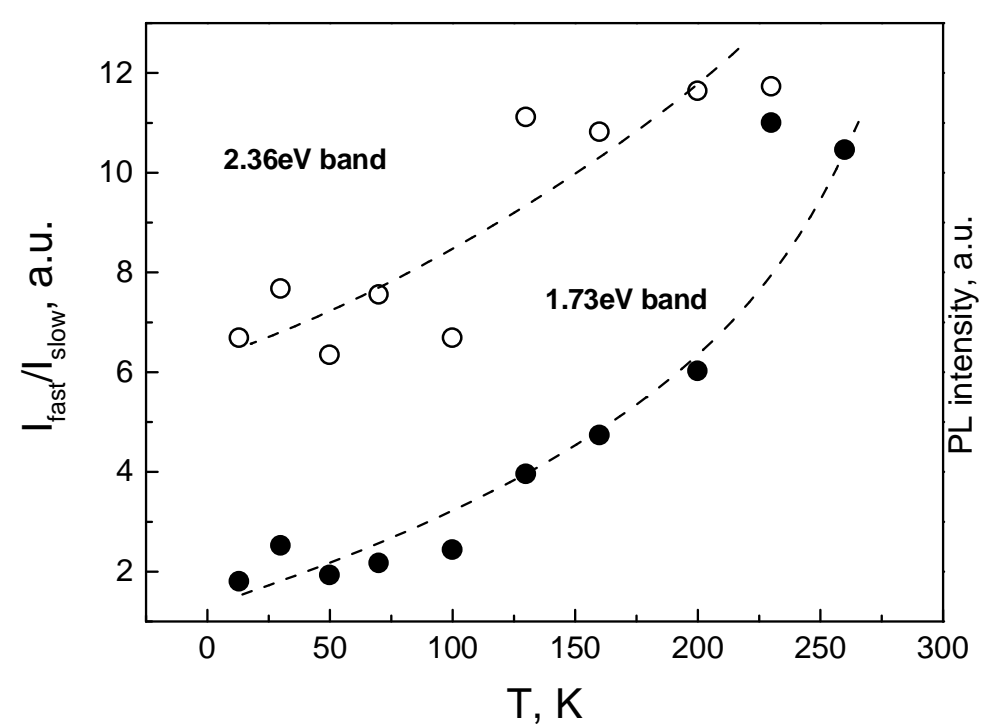

a)

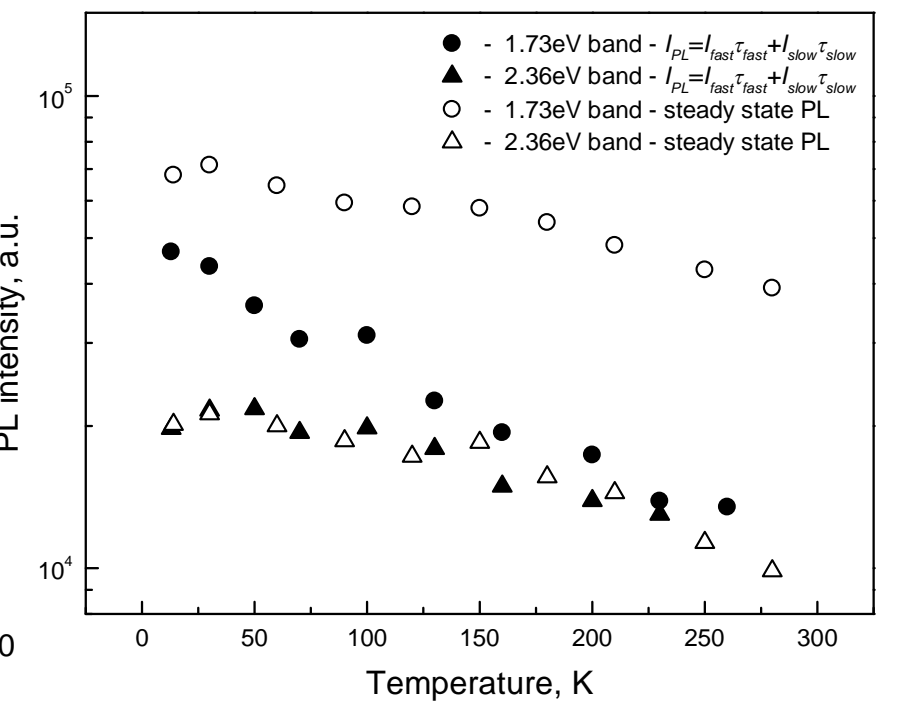

b)

Fig. $5-$ a) Variation of the ratio between fast and slow decay components $\left(I_{\text {fast }} / I_{\text {slow }}\right)$ in PL transients. Dependences for the red $(1.73 \mathrm{eV})$ and yellow $(2.36 \mathrm{eV})$ luminescence bands are shown by the solid and open symbols respectively. b) Temperature dependence of steady state PL intensity and its extrapolation through the fast and slow recombination components (in arbitrary units). The data are analyzed for the red $(1.73 \mathrm{eV})$ and yellow $(2.36 \mathrm{eV})$ luminescence bands.

\section{References}

[1] P.G. Kik, A. Polman, in Towards the First Silicon Laser, edited by L. Pavesi, S. Gaponenko and L. Dal Negro, Kluwer Academic Publishers 2003, p. 383.

[2] M. F. Cerqueira, M. Stepikhova, M. Losurdo, M.M. Giangregorio, E. Alves, T. Monteiro, M.J. Soares, C. Boemare, Microel. Journ., 34 (2003) 375.

[3] M.F. Cerqueira, M. Andritschky, L. Rebouta, J. A. Ferreira, M.F. da Silva, Vacuum 46 (1995) 1385.

[4] M. Stepikhova, M.F. Cerqueira, M. Losurdo, M.M. Giangregorio, E. Alves,T. Monteiro, M.J. Soares, Physics of the Solid State, in print

[5] M. Losurdo, M. F. Cerqueira, M.V. Stepikhova, E. Alves, M. Giangregorio, P. Pinto and J. A. Ferreira, Physica B 308-310, p.374 (2001) 
[6] M. Losurdo, M.F. Cerqueira, E. Alves, M. V. Stepikhova, M.M. Giangregorio, G. Bruno, Physica E Vol 16, pp .414-419 (2003)

[7] M. Stepikhova, W. Jantsch, G. Kocher, L. Palmetshofer, M. Schoisswohl and H.J. von Bardeleben. Appl. Phys. Lett. 71, 2975 (1997)

[8] M. F. Cerqueira, M. V. Stepikhova, M. Losurdo, M.M. Giangregorio, E. Alves, T. Monteiro, MJ Soares, C. Boemare, Microelectronics Journal 34, pp. 375-378 (2003)

[9] M. Fujii, M. Yoshida, S. Hayashi and K. Yamamoto, J. Appl. Phys. 84, 4525 (1998)

[10] T. Takagahara, K. Takeda, Phys. Rev. B46, 23, 15578 (1992)

[11] J. Valenta, R. Juhasz, J. Linnros, Appl. Phys. Lett., 80 (2002) 1070

[12] A. Polman, R.G. Elliman, in Towards the First Silicon Laser, edited by L. Pavesi,

S. Gaponenko and L. Dal Negro, Kluwer Academic Publishers 2003, p.209

[13] P.D.J. Calcott, K. J. Nash, L. T. Canham, M. J. Kane, D. Brumhead, J. Phys. C: Cond. Matt., 5 (1993) L91 